\section{Rec $\mathrm{C}_{\mathrm{A}}$}

Revista Colombiana de Ciencia Animal ISSN: 2027-4297

reciaeditor@unisucre.edu

Universidad de Sucre

Colombia

\title{
Caracterización de la macrofauna del suelo en fragmentos forestales en el municipio de Leticia, Amazonía colombiana
}

Tapia-Coral, Sandra Celia; Pinto Hernández, Carlos Augusto; Candre Iguedama, Adrian; Asencio, Carolina; Cuellar C, Reinaldo; Waldez, Fabiano

Caracterización de la macrofauna del suelo en fragmentos forestales en el municipio de Leticia, Amazonía colombiana

Revista Colombiana de Ciencia Animal, vol. 11, núm. 1, 2019

Universidad de Sucre, Colombia

DOI: https://doi.org/10.24188/recia.v11.n1.2019.690

Los autores permiten a RECIA reimprimir el material publicado en él. En caso de que un autor quiera traducir o usar una publicación parcial o completa de nuestro Diario, el autor debe obtener un permiso por escrito del editor de la revista.

Esta obra está bajo una Licencia Creative Commons Atribución 4.0 Internacional. 


\section{Caracterización de la macrofauna del suelo en fragmentos forestales en el municipio de Leticia, Amazonía colombiana}

Characterization of the soil macrofauna in forest fragments in the municipality of Leticia, Colombian amazon

Sandra Celia Tapia-Coral

Centro para la Biodiversidad y el Turismo del Amazonas,

SENA Regional Amazonas, Colombia

stapia@sena.edu.co

(iD http://orcid.org/0000-0003-0689-5485

DOI: https://doi.org/10.24188/recia.v11.n1.2019.690

Carlos Augusto Pinto Hernández

Centro Agroindustrialy de Fortalecimiento Empresarial,

SENA Regional Casanare, Colombia

cpintoh@sena.edu.co

(iD) http://orcid.org/0000-0001-7238-1306

Adrian Candre Iguedama

SENA Regional Amazonas, Colombia

adrian.candre@gmail.com

(DD http://orcid.org/0000-0003-3590-0941

Carolina Asencio

SENA Regional Amazonas, Colombia

karolays20-16@hotmail.com

(iD http://orcid.org/0000-0001-7088-8840

Reinaldo Cuellar C

SENA Regional Amazonas, Colombia

cimtar01@gmail.com

(iD http://orcid.org/0000-0002-9196-466X

Fabiano Waldez

Instituto Federal del Amazonas - IFAM Campus

Tabatinga, Brasil

fw.ifam@gmail.com

(DD http://orcid.org/0000-0003-4390-9399

\section{Resumen:}

La caracterización y la abundancia de la macrofauna del suelo y su relación con la morfología del suelo, fueron evaluadas en áreas de fragmentos forestales de bosques secundarios en el Centro para la Biodiversidad y el Turismo del Amazonas, del Servicio Nacional de Aprendizaje - SENA, en Leticia, Amazonía colombiana. La macrofauna del suelo (invertebrados mayores o iguales a $2 \mathrm{~mm}$ de diámetro) fue evaluada en dos períodos estacionales (mayo y octubre) con diferentes registros de precipitaciones. Fue encontrado una abundancia total de 5332 individuos de la macrofauna del suelo, distribuidos en 14 órdenes taxonómicas. El fragmento forestal conservado (FFC) fue el área que registró una mayor diversidad florística, y presentó la mayor densidad de la macrofauna del suelo en ambos periodos de muestreo. Los grupos de insectos Isoptera y Formicidae, fueron predominantes, sobre todo, en el FFC. Las comunidades de macrofauna del suelo no detectaron correlaciones significativas con las variables de la morfología del suelo en los

\section{NotAS DE AUTOR}


fragmentos forestales. Sin embargo, esa correlación se mostró significativa entre los dos periodos de muestreo, indicando que la estacionalidad de la pluviosidad puede afectar la densidad de la macrofauna del suelo dependiendo del periodo de muestreo.

Palabras Clave: Bosque secundario, ingenieros del suelo, macroinvertebrados del suelo, morfología del suelo.

\section{Abstract:}

The characterization and the abundance of the soil macrofauna and its relation with the morphology of the soil, were evaluated in areas of forest fragments of secondary forests in the Center for Biodiversity and Tourism of the Amazon, of the National Service of Learning - SENA, in Leticia, Colombian Amazon. The soil macrofauna (invertebrates greater than or equal to $2 \mathrm{~mm}$ in diameter) was evaluated in two seasoning periods (May and October) with different rainfall records. A total abundance of 5995 individuals of the soil macrofauna was found, distributed in 14 taxonomic orders. The conserved forest fragment (FFC) was the area that registered the highest floristic diversity, and presented the highest density of the soil macrofauna in both sampling periods. The groups of insects Isoptera and Formicidae were predominant, especially in the FFC. The soil macrofauna communities did not detect significant correlations with the soil morphology variables in the forest fragments. However, this correlation was significant between the two sampling periods, indicating that the seasonality of the rainfall may affect the density of the soil macrofauna depending on the sampling period.

KEYWORDS: Secondary forest, soil engineers, soil macroinvertebrates, soil morphology.

\section{INTRODUCCIÓN}

La Amazonía colombiana con aproximadamente 45\% del territorio, alberga cerca de 6500 especies vegetales y es considerada como una de las regiones con mayor diversidad del país (1). Sin embargo, la ampliación de la frontera agrícola, la ganadería extensiva, los incendios forestales y la tala selectiva para la venta de madera, constituyen los principales motores de la deforestación de bosques tropicales en el mundo, siendo que, en Colombia, se suman otras dinámicas, como la colonización y el desplazamiento de poblaciones, la minería y la siembra de cultivos ilícitos que aumentan la presión continua sobre el bosque (2). Un estudio realizado por el Sistema de Monitoreo de Bosques, del Instituto de Hidrología, Meteorología y Estudios Ambientales - IDEAM, establecieron un total de 140.356 hectáreas deforestadas en 2014 (3).

Una de las principales consecuencias de la deforestación es la creación de paisajes fragmentados con tamaños y hábitats completamente transformados (4), alterando el microclima del bosque y a la vez la extinción de muchas especies, tanto a nivel local como regional (5). La fragmentación de los bosques contribuye a la pérdida de hábitats y al aislamiento de pequeñas poblaciones naturales e incrementa las probabilidades de extinción y pérdida de la biodiversidad (6). Es así que el monitoreo de grupos de organismos claves para los ecosistemas de bosques tropicales es una estrategia que ha sido utilizada para intentar entender el impacto de las alteraciones ambientales a largo plazo (7). En este sentido, la macrofauna del suelo (invertebrados mayores o iguales que $2 \mathrm{~mm}$ de diámetro) representa un grupo funcional que regula importantes procesos ecosistémicos en el suelo, como la descomposición de la materia orgánica, la formación de estructuras biogénicas y el reciclaje de nutrientes (8), y junto con la agregación del suelo, determinada a través de su morfología (atributos biológicos y físicos), se encuentran fuertemente relacionada con sus propiedades, como la infiltración y el almacenamiento de agua y carbono (9).

El objetivo principal de este estudio fue evaluar la composición de la comunidad de la macrofauna del suelo y su relación con la morfología del suelo en fragmentos forestales de bosques secundarios, con la finalidad de levantar informaciones que puedan auxiliar en el monitoreo de las alteraciones en los paisajes naturales con el crecimiento urbano.

\section{MATERIALES Y MÉTODOS}

Área de estudio: El trabajo fue realizado en tres áreas de fragmentos forestales de bosque secundario denominados para este estudio como: (i) fragmento forestal de borda (FFB), cercano a la carretera; (ii) 
fragmento forestal conservado (FFC), con mayor diversidad de especies arbóreas; (iii) fragmento forestal de transición (FFT), localizado en un área abierta cercana de una quebrada y (iv) un área alterada de pastizal (AP). Todos los paisajes estudiados se encontraron localizados en el Centro para la Biodiversidad y el Turismo del Amazonas del Servicio Nacional de Aprendizaje - SENA Regional Amazonas, en el Municipio de Leticia, Amazonas, Colombia (Figura 1).
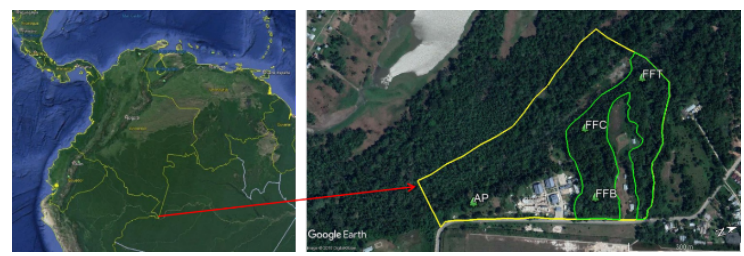

FIGURA 1

Localización del área de estudio

En el extremo sur del departamento de Amazonas, mostrando los fragmentos evaluados: fragmento forestal de borda (FFB), fragmento forestal conservado (FFC), fragmento forestal de transición (FFT) y área de pastizal (AP) en Centro para la Biodiversidad y el Turismo del Amazonas, SENA Regional Amazonas, Leticia, Amazonas, Colombia. Fuente: Google Maps, adaptado.

Autor

La vegetación predominante en los fragmentos forestales fue típica de bosque secundario en estado de regeneración, con enriquecimiento de especies arbóreas nativas de la región y el área alterada de pastizal fue cubierta por pasto de Brachiaria sp. (10) (Tabla 1).

TABLA 1

Localización geográfica y descripción general de los fragmentos forestales y área de pastizal evaluados en el Centro para la Biodiversidad y el Turismo del Amazonas, Leticia, Amazonas.

\begin{tabular}{|c|c|c|}
\hline $\begin{array}{l}\text { Fragmentos } \\
\text { Forestales }\end{array}$ & $\begin{array}{c}\text { Coordenadas } \\
\text { Geográficas }\end{array}$ & $\begin{array}{l}\text { Principales especies de la } \\
\text { composición florística (10) }\end{array}$ \\
\hline $\begin{array}{l}\text { Fragmento } \\
\text { Forestal de } \\
\text { Borda - FFB }\end{array}$ & $\begin{array}{l}\text { W } 69^{\circ} 56^{\circ} 46 \\
23^{\prime \prime} \mathrm{S} 04^{\circ} \\
11^{\prime} 51.61^{\prime \prime}\end{array}$ & $\begin{array}{l}\text { Bellucia pentamera Naudin } \\
\text { Cecropia ficifolia Warb. ex Snethl. } \\
\text { Mauritia flexuosa L.f. } \\
\text { Senna silvestris (Vell.) H.S.Irwin \& } \\
\text { Barneby }\end{array}$ \\
\hline $\begin{array}{l}\text { Fragmento } \\
\text { Forestal } \\
\text { Conservado - } \\
\text { FFC }\end{array}$ & $\begin{array}{l}\text { W } 69^{\circ} 56^{\prime} 52 \\
25^{\prime \prime} \mathrm{S} 04^{\circ} \\
11^{\prime} 50.74^{\prime \prime}\end{array}$ & $\begin{array}{l}\text { Astrocaryum chambira Burret } \\
\text { Foramea multiflora DC. } \\
\text { Inga edulis Mart. } \\
\text { Inga sertulifera DC. } \\
\text { Miconia serrulata (DC) Naudin } \\
\text { Senna silvestris (Vell.) H.S.Irwin \& } \\
\text { Barneby } \\
\text { Swartzia polyphylla DC. }\end{array}$ \\
\hline $\begin{array}{l}\text { Fragmento } \\
\text { Forestal de } \\
\text { Transición - } \\
\text { FFT }\end{array}$ & $\begin{array}{l}\text { W } 69^{\circ} 56^{\prime} 55 \\
09^{\prime \prime} 504^{\circ} \\
11^{\prime} 44.58^{\prime \prime}\end{array}$ & $\begin{array}{l}\text { Alchornea latifolia Sw. } \\
\text { Bellucia pentamera Naudin } \\
\text { Miconia radulifolia } \\
\text { (Benth.) Naudin } \\
\text { Vismia macrophylla Kunth }\end{array}$ \\
\hline $\begin{array}{l}\text { Area de } \\
\text { Pastizal (AP) }\end{array}$ & $\begin{array}{l}\text { W } 69^{\circ} 56^{\circ} 48 \\
82^{\prime \prime} \mathrm{S} 04^{\circ} \\
12^{\prime} 01.97^{\prime \prime}\end{array}$ & Brachiaria sp. \\
\hline
\end{tabular}

Autor

Toma de muestras: Los muestreos de la macrofauna del suelo fueron realizados durante los meses de mayo y octubre de 2017, y de acuerdo con el IDEAM 2017 (11) se registró una precipitación mensual de 435 $\mathrm{mm}$ con una temperatura promedio de $27,17^{\circ} \mathrm{C}$ en mayo y una precipitación mensual de $137 \mathrm{~mm}$ con una temperatura promedio de $27,29^{\circ} \mathrm{C}$ en octubre (Figura 2). 


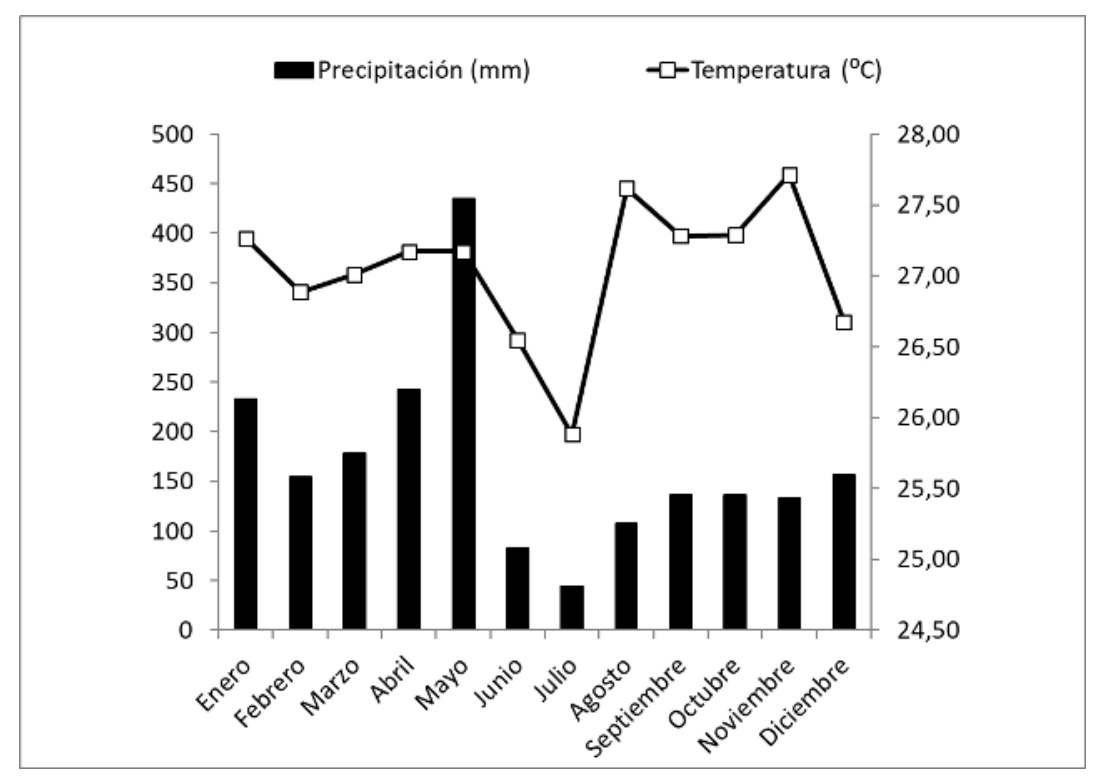

FIGURA 2

Variación de la precipitación pluvial ( $\mathrm{mm}$ ) y temperatura promedio mensual (oC) referente al año 2017, municipio de Leticia, Amazonas, Colombia. Fuente: www.ideam.gob.co (adaptado para este estudio).

Autor

El diseño experimental fue el recomendado por el protocolo detallado de muestreo de la macrofauna edáfica del proyecto Sustainable Amazonian Landscape del Centro Internacional de Agricultura Tropical (CIAT) en Palmira, Colombia (12).

La macrofauna del suelo fue evaluada con la metodología del programa de Tropical Soil Biology and Fertility (TSBF) (13), adecuado para este estudio hasta una profundidad de $20 \mathrm{~cm}$. En cada una de las áreas se retiraron seis monolitos (bloques de suelo) de $25 \times 25 \mathrm{~cm}$ de lado y una profundidad de $20 \mathrm{~cm}$ donde fueron establecidos tres estratos: hojarasca sobre el suelo y dos niveles de profundidad de $0-10 \mathrm{~cm}$ y $10-20 \mathrm{~cm}$.

La macrofauna del suelo fue separada en el campo y conservada en frascos con alcohol al $96 \%$. Posteriormente, en el laboratorio, los animales fueron identificados a nivel de grandes grupos taxonómicos (14) (Figura 3).

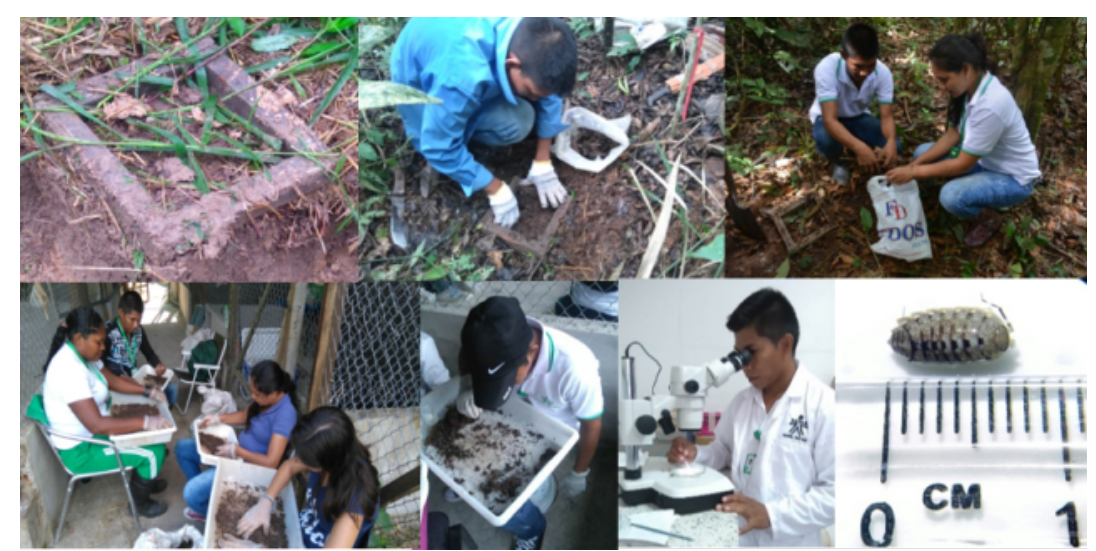

FIGURA 3

Secuencia del muestreo de la macrofauna del suelo, separación e identificación en campo (mayo y octubre, 2017) y proceso de identificación en el laboratorio (noviembre, 2017). 
La morfología del suelo fue evaluada según la metodología descrita por Velásquez et al (15) y los muestreos del suelo fueron realizados utilizando un cuadro de metal de $10 \times 10 \mathrm{~cm}$ de lado y $10 \mathrm{~cm}$ de profundidad en los mismos puntos donde fue muestreada la macrofauna.

El proceso de separación de los agregados del suelo fue realizado en el laboratorio, siendo que los atributos fueron separados en sus diversos componentes: (i) agregados biogénicos producidos por las lombrices, termitas, hormigas y algunas larvas de coleópteros, los cuales pueden diferenciarse por sus colores oscuros (deyecciones de lombrices principalmente), formas circulares, galerías y macroporos; (ii) agregados físicos productos de compactaciones producidas por el pisoteo de animales y también, de la acción del agua, formados generalmente en suelos arcillosos y se diferencian por sus formas planas y angulares; (iii) agregados producidos por las raíces de las plantas; (iv) materia orgánica (fragmentos de tallos, hojas, madera, semillas, flores, etc.); (v) suelo sin agregar, donde el material residual de la separación de los componentes es tamizado (0.5cm de diámetro); (vi) raíces de plantas y (vii) piedras (15).

Después de separados, los diversos agregados componentes del suelo fueron secados a temperatura ambiente por 72 horas y pesados en una balanza gravimétrica digital (Figura 4) (16).

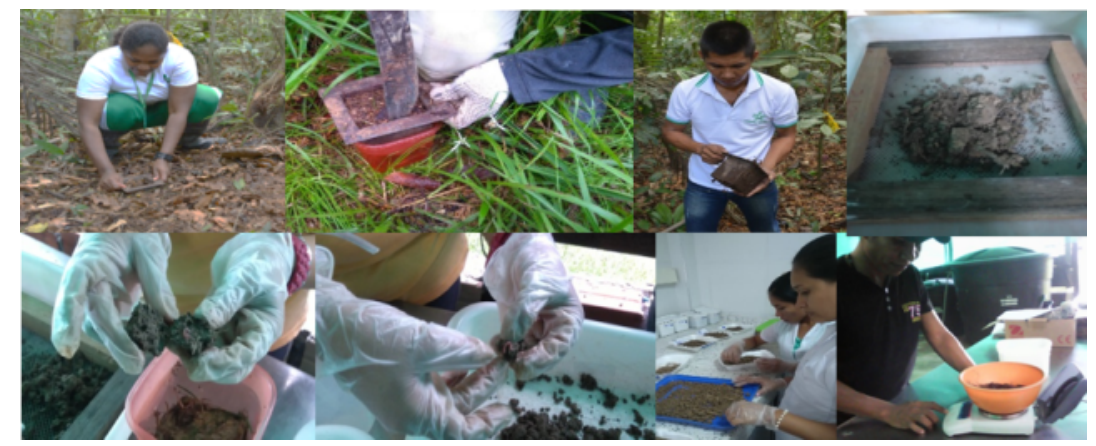

FIGURA 4

Secuencia del muestreo de la morfología del suelo y proceso de separación de los agregados del suelo en el laboratorio (mayo y octubre 2017).

Fotos: Carolina Asencio y Sandra Celia Tapia Coral.

Análisis de los datos: Para evaluar el efecto de los diferentes períodos estacionales en que fueron realizados los muestreos de la macrofauna del suelo, fue realizado un análisis estadístico descriptivo seguido de la prueba t de Student con las probabilidades corregidas por la prueba de Bonferroni (17) en el programa Systat 12 (18).

También, se realizó un análisis estadístico multivariado de componentes principales (ACP) con la prueba de Montecarlo (17), conforme metodología indicada en varios estudios de investigación (9,19, 20, 21, 22). Estos análisis son realizados con el propósito de identificar los factores predominantes para las variables de las comunidades de la macrofauna del suelo (densidad ind $/ \mathrm{m} 2$ ) y de la morfología del suelo (tipos de atributos) en los fragmentos forestales y en el área de pastizal. Un análisis estadístico de coinercia fue realizado para determinar correlaciones entre los vectores del análisis de componentes principales (ACP) para las variables de la macrofauna del suelo y de la morfología del suelo en las áreas estudiadas en ambos periodos de muestreo. Los análisis estadísticos multivariados fueron realizados en el software R (23) y el ACP en el paquete ADE4 (24).

\section{Resultados Y Discusión}

Macrofauna del suelo: Fueron encontrados un total de 5332 individuos de la macrofauna del suelo distribuidos en un total de 14 grupos taxonómicos, siendo que en mayo (2329 individuos, distribuidos en 12 órdenes taxonómicas), y en octubre (3003 individuos, distribuidos en 14 órdenes taxonómicas) (Tabla 2). 
TABLA 2

Abundancia de los grupos taxonómicos de la macrofauna del suelo (individuos) en mayo y octubre de 2017, en el fragmento forestal de borda (FFB), fragmento forestal conservado (FFC), fragmento forestal de transición (FFT) y área de pastizal (AP).

\begin{tabular}{lllllllll}
\hline & \multicolumn{9}{l}{ Mayo } & \multicolumn{7}{l}{ Octubre } \\
Grupos & FFB & FFC & FFT & AP & FFB & FFC & FFT & AP \\
\hline Aranea & 7 & 9 & 8 & 10 & 13 & 25 & 9 & 2 \\
Blataria & 4 & 3 & 1 & 4 & 14 & 4 & 0 & 2 \\
Coleoptera & 60 & 111 & 83 & 66 & 14 & 12 & 12 & 16 \\
Chilopoda & 4 & 8 & 4 & 5 & 3 & 16 & 13 & 6 \\
Diplopoda & 11 & 16 & 17 & 33 & 4 & 7 & 15 & 3 \\
Formicidae & 102 & 175 & 168 & 52 & 562 & 1066 & 350 & 12 \\
Gastropoda & 4 & 0 & 1 & 12 & 1 & 7 & 1 & 4 \\
Isopoda & 16 & 14 & 9 & 52 & 6 & 10 & 19 & 13 \\
Homoptera & 0 & 0 & 0 & 0 & 0 & 1 & 0 & 0 \\
Isoptera & 20 & 680 & 197 & 34 & 223 & 82 & 143 & 26 \\
Larvas & 7 & 8 & 8 & 2 & 9 & 14 & 10 & 10 \\
Oligochaeta & 87 & 11 & 74 & 121 & 79 & 4 & 30 & 107 \\
Orthoptera & 0 & 0 & 0 & 0 & 4 & 1 & 9 & 0 \\
Pseudoescorpion & 0 & 5 & 5 & 0 & 0 & 10 & 1 & 0 \\
Total & 323 & 1040 & 575 & 391 & 932 & 1259 & 611 & 201 \\
\hline
\end{tabular}

Autor

La abundancia total de individuos registrados en los dos períodos de muestreo no presento diferencia significativa $[\mathrm{t}=-0,75 ; \mathrm{GL}=71 ; \mathrm{p}$ (Bonferroni) $=0,45]$, siendo en promedio, relativamente menor en mayo $(\bar{x}=40,6)$ que en octubre $(\bar{x}=46,9)$. Cuando analizamos la abundancia total de individuos en los fragmentos forestales y en el área de pastizal, entre los dos períodos de muestreo, fue encontrado una diferencia significativa únicamente para el pastizal $\left[\mathrm{t}=2,19 ; \mathrm{GL}=17 ; \mathrm{p}_{\text {(Bonferroni) }}=0,04\right]$, siendo en promedio, mayor en mayo $(A P \bar{x}=24,66)$ que en octubre $(A P \bar{x} 12,55)$. Para los fragmentos forestales no fueron encontrados diferencias significativas.

La riqueza total de los grupos taxonómicos registrados en los dos períodos de muestreo, no tuvo diferencia significativa $\left[\mathrm{t}=1,51 ; \mathrm{GL}=71 ; \mathrm{p}_{\text {(Bonferroni })}=0,13\right]$, siendo que el promedio fue relativamente mayor en mayo $(\bar{x}=4,36)$ que en octubre $(\bar{x}=3,87)$. La riqueza de grupos taxonómicos de la macrofauna del suelo observada en este estudio (14 grupos taxonómicos) fue similar al reportado por Marín et al (25), que encontraron 15 grupos en sistemas de labranzas en el Valle del Cauca. Entre tanto, Botina et al (26) reportó 10 grupos en sistemas de labranza de papa en el departamento de Pasto y Suarez et al (21) reportaron 10 grupos en sistemas agroforestales en el Caquetá, Amazonía colombiana. No en tanto, Duran et al (27) reportaron hasta 21 grupos taxonómicos de la macrofauna del suelo en sistemas agroforestales con diferentes composiciones también en el Caquetá, Amazonia colombiana. Los estudios realizados en Colombia fueron enfocados en sistemas de chagras, agroforestales y cultivos con diferentes arreglos forestales en su composición $(19,21,27)$. En la parte sur de la Amazonía colombiana aún son escasos los estudios de la macrofauna del suelo (16), siendo el presente estudio uno de los pocos realizados en áreas de fragmentos forestales de bosques secundarios.

Con relación a la distribución vertical de la densidad de la macrofauna del suelo, los datos demostraron que en el mes de mayo hubo una distribución de invertebrados predominante en la capa de hojarasca y sobre todo, en el primer horizonte del suelo $(0-10 \mathrm{~cm})$ en todos los fragmentos forestales y el área de pastizal (Figura 5). Indicando que durante el periodo más húmedo los primeros horizontes del suelo tienen una mayor contribución como fuente de energía y nutrientes (28).

El segundo horizonte del suelo $(10-20 \mathrm{~cm})$ presentó una densidad muy baja de invertebrados en todas las áreas y en los dos periodos de muestreo, lo que puede indicar que por debajo de los $10 \mathrm{~cm}$ de profundidad hay una menor contribución de la actividad de la macrofauna del suelo en este horizonte. 


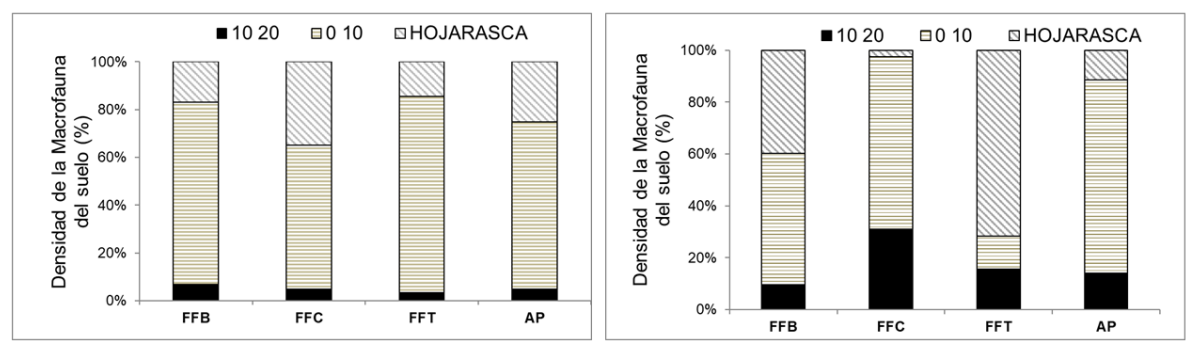

\section{FIGURA 5}

Distribución vertical de la macrofauna del suelo (\%) en el fragmento forestal de borda (FFB),

fragmento forestal conservado (FFC), fragmento forestal de transición (FFT) y el área de pastizal (AP) en los dos periodos de muestreo en mayo (izquierda) y octubre (derecha) de 2017.

Resultados similares a los observados en este estudio sobre la distribución vertical de la densidad de la macrofauna del suelo, fueron reportados para la Amazonía colombiana (27) y en plantíos forestales de la Amazonía peruana (29), con una mayor actividad de la macrofauna reflejada en los primeros horizontes del suelo y en la capa de hojarasca.

En octubre, durante el periodo de menor humedad, la macrofauna del suelo tuvo una mayor predominancia en el fragmento forestal conservado (FFC) y el fragmento forestal de borda (FFB) (Figura 5). Sin embargo, el fragmento forestal de transición (FFT) y el área de pastizal (AP) tuvieron una disminución considerable de la macrofauna del suelo en este período de muestreo (Figura 5), lo que puede indicar que la macrofauna fue afectada por las menores precipitaciones en este período, principalmente en el pastizal por la falta de cobertura del suelo. Resultados similares fueron reportados en otros estudios donde la comunidad de la macrofauna del suelo generalmente fue afectada en periodos con menores humidad $(20,21,27)$.

En el fragmento forestal conservado (FFC) fue observada la mayor densidad de la macrofauna del suelo en los dos períodos de muestreos, lo que puede estar relacionado con la mayor diversidad vegetal de especies arbóreas en este fragmento $(10)$. Otros autores $(28,30)$ reportaron que la diversificación de plantas ofrece una mayor diversidad de microhábitats y de esta manera contribuyen a una mayor diversidad y densidad biológica del suelo.

Las lombrices de tierra (Oligochaeta) y los grupos de insectos sociales, hormigas (Formicidade) y termitas (Isoptera), fueron los más predominantes en el FFC y FFT (Tabla 2). Estos tres grupos de la macrofauna del suelo son clasificados como "ingenieros del ecosistema" (8) porque producen estructuras biológicas originadas de sus actividades, que promueven alteraciones en los atributos micromorfológicos y físicos del suelo, como la infiltración de agua y la aireación del suelo por el aumento de la porosidad. Sin embargo, estos grupos son muy sensibles a las variaciones del uso de la tierra y de la cobertura del suelo y, por lo tanto, pueden ser estudiados como indicadores de recuperación del suelo de bosques forestales (19, 31).

Las termitas son organismos oportunistas y resistentes a las perturbaciones inducidas, que indican hábitats menos conservados o con algún nivel de degradación (32). En los fragmentos forestales los géneros de termitas (Isoptera) predominantes fueron: Anoplotermes sp. Cylindrotermes sp. y Nasusitermes sp.

La lombriz exótica Pontoscolex corethrurus fue predominante en todos los fragmentos forestales e inclusive en el área de pastizal. Otros estudios, también ya mostraron la predominancia de P. corethrurus en ambientes con un cierto grado de perturbación o manejo del suelo $(29,33)$ y por lo tanto, esta lombriz es resistente y frecuente en ambientes donde el grado de perturbación es intensivo como es el caso de los pastizales.

En el segundo período de muestreo (octubre) las hormigas (Formicidae) fueron muy presentes en los fragmentos forestales, principalmente en el bosque con mayor diversidad de árboles (FFC), y poco frecuentes en el área de pastizal (Tabla 2). Las condiciones climáticas y el alimento disponible debido a la cobertura de hojarasca del suelo (20) en los fragmentos forestales, probablemente determinó una mayor actividad de las 
hormigas, indicando el estado de degradación del pastizal para los organismos edáficos, más afectados debido a la falta de cobertura del suelo (33).

El análisis de componentes principales $(\mathrm{ACP})$ presentó una diferencia significativa $(\mathrm{p}=0,002)$ en la separación de los fragmentos forestales y el área de pastizal en función de los indicadores de la macrofauna del suelo. El primer factor explicó el 22\% de la variabilidad de los datos y separó principalmente el FFC de las demás áreas estudiadas. El segundo factor explicó el 18\% y separó el FFT y el AP del FFC y el FFB (Figura 6).

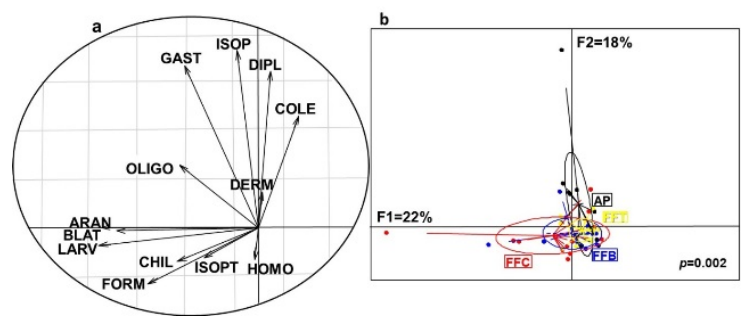

FIGURA 6

Análisis de componentes principales (PCA) de los indicadores de la macrofauna del suelo. (a) circulo de correlación de las variables: ARAN (Araneae), BLAT (Blattodea), CHIL (Chilopoda), COLE (Coleoptera), DERM (Dermaptera), DIPL (Diplopoda), FOR (Formicidae), GAST (Gasteropoda), ISOP (Isopoda), ISOPT (Isoptera), LARV (larvas), OLIGO (Oligochaeta). (b) Proyección de los fragmentos forestales: FFB (fragmento forestal de borda), FFC (fragmento forestal conservado), FFT (fragmento forestal de transición) y AP (área de pastizal) en función de los indicadores. Autor

El ACP también presento diferencia significativa $(\mathrm{p}=0,001)$ de las variables de la macrofauna del suelo entre los dos periodos de muestreo (mayo y octubre) (Figura 7), indicando que la comunidad de la macrofauna del suelo es sensible a los cambios de precipitación en una corta escala de tiempo, como ya fue observado en otros estudios $(20,21,32)$.

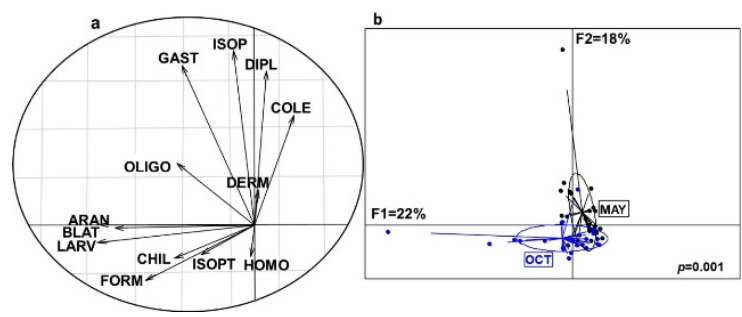

FIGURA 7

Análisis de componentes principales (PCA)

(a) Círculo de correlación de las variables de los indicadores de la macrofauna del suelo (b) Proyección de los periodos de muestreo mayo (MAY) y octubre (OCT) en función de los indicadores de la macrofauna del suelo.

Morfología del suelo. En el muestreo realizado en mayo, la composición de agregados de la materia orgánica del suelo (MO) (\%) fue la más abundante en el FFC. Los agregados biológicos (AB) fueron relativamente similares en todos los fragmentos forestales muestreados y el área de pastizal en los dos periodos de muestreo. Sin embargo, en octubre se encontró una mayor cantidad de AB en el FFC y FFB; y mayores proporciones de los agregados físicos (AF) en todos los fragmentos forestales y el AP (Figura 8). 

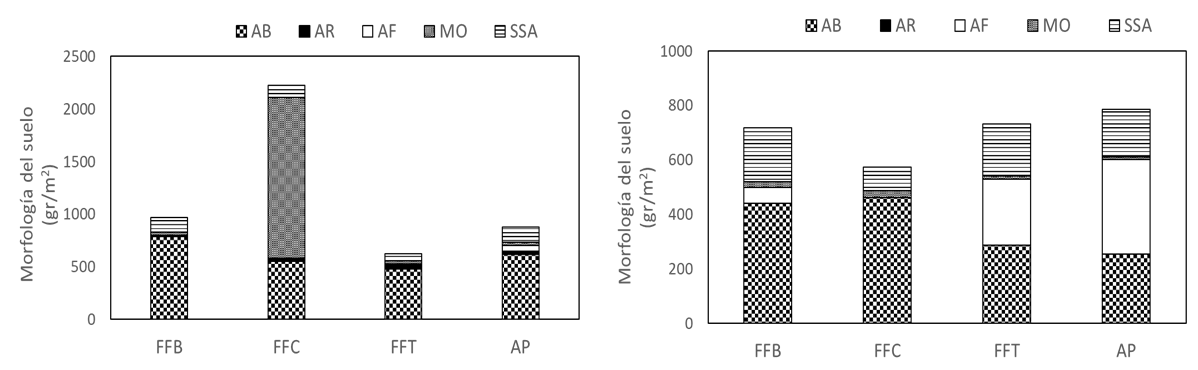

FIGURA 8

Composición de la morfología del suelo (grm2) en agregados biológicos $(\mathrm{AB})$, agregados de raíz (AR), agregados físicos (AF), materia orgánica (MO) y suelo sin agregar (SSA) en el fragmento forestal de borda (FFB), fragmento forestal conservado (FFC), fragmento forestal de transición (FFT) y el área de pastizal (AP) en ambos periodos de muestreo, mayo (izquierda) y octubre (derecha).

$\mathrm{Al}$ analizar las variables de la morfología del suelo por medio del ACP, fue explicada un total de $58.2 \%$ de la variación en los dos primeros factores. Sin embargo, no hubo diferencia significativa entre las áreas de estudio evaluadas. Entre tanto, cuando analizamos las variables de la morfología del suelo entre los dos periodos de muestreo (mayo y octubre) encontramos una diferencia significativa $(\mathrm{p}=0.001)$ (Figura 9).

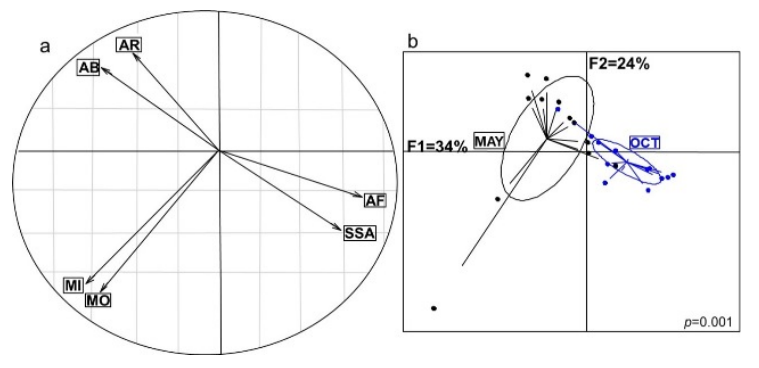

FIGURA 9

Análisis de componentes principales (PCA)

(a) Círculo de correlación de las variables de los indicadores de la morfología del suelo de agregados biológicos (AB), agregados de raíz (AR), agregados físicos (AF), materia orgánica (MO) y suelo sin agregar (SSA) y (b) Proyección de los periodos de muestreo mayo (MAY) y octubre (OCT) en función de los indicadores de la morfología del suelo. Autor

El análisis estadístico de coinercia no detecto correlaciones significativas entre las variables de la macrofauna del suelo y de la morfología del suelo, en los fragmentos forestales y en el área de pastizal. Sin embargo, nuestros datos demostraron diferencias na composición de la macrofauna entre los dos periodos estacionales de muestreo. El estudio de Duran et al (27) mencionó que la estructura de la comunidad de la macrofauna del suelo varía de acuerdo con el periodo de muestreo, siendo que las mínimas precipitaciones en sistemas agroforestales, resultan en una abundante capa de hojarasca en el suelo, que proporciona refugio y alimento para los macroinvertebrados. También, la densidad y diversidad de la macrofauna es afectada entre otros factores, por la composición de los sistemas (21). Sin embargo, el FFC fue el que presento mayores densidades de la macrofauna del suelo en ambos periodos de muestreo, y mayores cantidades de agregados biológicos, siendo estos $\mathrm{AB}$ relacionados con una mayor actividad biológica y, sobre todo, con la acción de los ingenieros del ecosistema (31).

Consideraciones finales. La macrofauna del suelo fue predominante en los primeros horizontes del suelo: hojarasca y $0-10 \mathrm{~cm}$ de profundidad en todos los fragmentos forestales evaluados, siendo que los grupos de insectos sociales, Isoptera y Formicidae, fueron los más predominantes en el FFC. Este fragmento presentó una mayor diversidad de composición de árboles y la mayor densidad de la macrofauna del suelo en ambos periodos de muestreo. Entre tanto, la diversidad de grupos de la macrofauna del suelo fue relativamente baja 
en los fragmentos de muestreo, lo que puede indicar que estas áreas tienen un cierto grado de perturbación. Por otrolado, estas áreas están en estado de regeneración forestal y, por lo tanto, son laboratorios permanentes para el desarrollo de investigaciones y prácticas de campo de los programas ambientales del centro del SENA Regional Amazonas. Resaltamos así, la importancia de los fragmentos forestales como fundamentales para el mantenimiento de la biodiversidad y de los servicios ecosistémicos del suelo, particularmente los fragmentos forestales urbanos, cada vez más afectados por el crecimiento de la ciudad de Leticia.

\section{Agradecimientos.}

Al Servicio Nacional de Aprendizaje (SENA) a través del Sistema de Investigación, Desarrollo Tecnológico e Innovación (SENNOVA), por el financiamiento del proyecto "Servicios ecosistémicos del suelo en fragmentos forestales naturales y manejados en Leticia, Amazonas" (SGPS-742-2016), donde este estudio hizo parte. Agradecimiento especial a los aprendices del semillero BioAmazonas de la Regional Amazonas, que participaron y colaboraron en el proyecto. Al Ing. MSc. Ervin Humprey Duran, por la identificación de los géneros de termitas.

\section{REFERENCIAS}

1. Rangel O. (Ed). Colombia: Diversidad biótica. Instituto de Ciencias Naturales. Universidad Nacional de Colombia. Bogotá. 1987.

2. CEPAL - Comisión Económica para América Latina y el Caribe y Patrimonio Natural. Amazonia posible y sostenible. Bogotá. 2013. [Citado 27 sep 2018] URL Disponible en: https://repositorio.cepal.org/ handle/11362/1506

3. IDEAM - Instituto de Hidrología, Meteorología y Estudios Ambientales. Aumenta deforestación en Colombia para 2015. [Citado 05 abril] URL Disponible en: http://www.ideam.gov.co

4. Kattan GH. Fragmentación: patrones y mecanismos de extinción de especies. En: Guariguata MR, Kattan GH. Editores. Ecología y conservación de Bosques Neotropicales. EULAC/GTZ. 1 ed. Ediciones LUR. Cartago, CR. p. 561-590. 2002.

5. Bennett A. Enlazando el paisaje: el papel de los corredores y la conectividad en la conservación de la vida silvestre. Cambridge, RU: UICN. p.276. 1998.

6. Gurrutxaga MSV, Lozano PJV. Efectos de la fragmentación de hábitats y pérdida de conectividad ecológica dentro de la dinámica territorial. Polígonos. Rev. de Geografía 2006; 16:35-54.

7. Butler RA, Laurance FW. New strategies for conserving tropical forests. Trends in Ecology \& Evolution 2008; 974. [Citado 20 august 2018] Available URL in: https://es.mongabay.com/2008/08/nuevas-estrategias-paraconservar-los-bosques-tropicales/

8. Lavelle P, Decaëns T, Aubert M, Barot S, Blouin M, Bureau F. et al. Soil invertebrates and ecosystem services. Eur J Soil Biol 2006; 42:S3-S15. DOI: 10.1016/j.ejsobi.2006.10.002

9. Lavelle P, Rodríguez N, Arguello O, Bernal J, Botero C, Chaparro P, et al. Soil ecosystem services and land use in the rapidly changing Orinoco River Basin of Colombia. Agric Ecosyst Environ. 2014; 185:106-117. DOI: https:// dx.doi.org/10.1016/j.agee.2013.12.020

10. Cuellar R, Candre IA, Quiñones GML, Tapia-Coral SC. Inventario de la vegetación en fragmentos forestales naturales en el Sena Regional Amazonas, Leticia, Amazonas, Colombia. En: UEA editores. Anais do $7^{\circ}$ Encontro Internacional de Ensino, e Pesquisa em Ciências na Amazônia. Tabatinga, Amazonas, Brasil. [Citado 05 abril 2018]. 2017.

11. IDEAM - Instituto de Hidrología, Meteorología y Estudios Ambientales. [Internet] 2017. [citado 05 abr 2017] URL Disponible en: http://www.ideam.gov.co/web/tiempo-y-clima/clima 
12. CIAT - Centro de Investigación en Agricultura Tropical. Sustainable Amazonian Landscapes Project. [Citado 05 abril 2018]. Disponible en: https://amazonlandscapes.org/ 2017.

13. Anderson JM, Ingram JS. Tropical soil biology and fertility: A handbook of methods. 2 ed., CAB International, Wallingford, UK. 256p. 1993.

14. Ruiz CN, Lavelle P, Jiménez JJ. Soil macrofauna Field Manual. FAO. 2008.

15. Velásquez E, Pelosi C, Brunet D, Grimaldi M, Martins M, Rendeiro AC, Barrios E. et al. This ped is my ped: visual separation and NIRS spectra allow determination of the origins of soil macro-aggregates. Pedobiologia 2007b; 51:75-87. DOI: 10.1016/j.pedobi.2007.01.002

16. Tapia-Coral SC, Candre IA, Pinto HCA. Cartilla para muestreo de macroinvertebrados del suelo. SENA editores. 2017.

17. Zar JH. Biostatistical analysis. 3rd edition. Prentice-Hall, Upper Saddle River, New Jersey, USA. 1996.

18. Wilkinson L. Systat: The system for statistics, version 12, Systat Software Inc. Evanston, Illinois, USA. 2007.

19. Velásquez E, Lavelle P, Andrade M. GISQ, a multifunctional indicator of soil quality. Soil Biol Biochem 2007a; 39:3066-3080. DOI: https://doi.org/10.1016/j.soilbio.2007.06.013

20. Lima SS, Aquino AM, Leite LFC, Velásquez E, Lavelle P. Relação entre a macrofauna edáfica e atributos químicos do solo em diferentes agroecossistemas. Pesq. Agropec. Bras. 2010; 45(3):322-331. DOI: http:// dx.doi.org/10.1590/S0100-204X2010000300013

21. Suarez SJC, Duran BEH, Rosas PG. Macrofauna edáfica asociada con sistemas agroforestales en la Amazonía Colombiana. Acta Agron. 2015; 64(3):214-220. DOI: http://dx.doi.org/10.15446/acag.v64n3.38033

22. Tapia-Coral SC, Teixeira LA, Velásquez E, Waldez F. Macroinvertebrados del suelo y sus aportes a los servicios ecosistémicos, una visión de su importancia y comportamiento. Rev Colombiana Cienc Anim. 2016; 8(Supl):260-267. DOI: https://doi.org/10.24188/recia.v8.n0.2016.380

23. R Development Core Team. R: A language and environment for statistical computing. R Foundation for Statistical Computing. Vienna, Austria. ISBN 3-900051-07-0. Disponible en: URL http://www.R-project.org/ 2014.

24. Dray S, Dufour AB. The ade4 package: implementing the duality diagram for ecologist. J Stat Softw. 2007; 22(4):1-20. DOI: 10.18637 /jss.v022.i04

25. Marín E, Feijoo A. Efecto de la labranza sobre macroinvertebrados del suelo en vertisoles de un área de Colombia. Terra Latinoamericana 2005; 25(3):297-310. DOI: http://www.chapingo.mx/terra/

26. Botina BG, Velásquez AI, Bacca T, Castillo JF, Díaz LG. Evaluación de la macrofauna del suelo en Solanum tuberosum (solanales: solanaceae) con sistemas de labranza tradicional y mínima. Bol. Cient. Mus. His. Nat. 2012; 16(2):69-77. ISSN 0123 - 3068.

27. Duran BEH, Suárez SJC. Fauna del suelo y hojarasca en arreglos agroforestales de la Amazonía Colombiana. Momentos de Ciencia. 2013; 10(1):59-66. URL Disponible en: https://www.udla.edu.co/revistas/index.php/ momentos-de-ciencia/article/view/253/0

28. Da Silva MK, Da Gama EF, Da Gama AC, Machado RC, Baligar VC. Soil and litter fauna of cacao agroforestry systems in Bahia, Brazil. Agrofor Syst 2009; 76:127-138. DOI: https://link.springer.com/article/10.1007/ s10457-008-9178-6

29. Tapia-Coral SC, Luizão F, Pashanasi B, Del Castillo D. Comunidade de macroinvertebrados do solo em plantios florestais e sistemas naturais na Amazônia peruana. Folia Amazónica 2012; 21(1-2):33-44. DOI: https:// doi.org/10.24841/fa.v21i1-2.30

30. Laossi KR, Barot S, Carvalho D, Desjardins T, Lavelle P, Martins M. Effects of plant diversity on plant biomass production and soil macrofauna in Amazonia pastures. Pedobiologia 2008; 51:397-407. DOI: 10.1016/ j.pedobi.2007.11.001

31. Da Silva E, Velásquez E, Santos A, Bartz MLC, Lavelle P, Brown GG. Indicador general de calidad del suelo en diferentes sistemas de uso del suelo en el Sur de Brasil. En: Memorias del V Congreso Latinoamericano de Agroecología. La Plata, Argentina. 2015: URL Disponible en: http://sedici.unlp.edu.ar/ bitstream/handle/10915/58826/Documento_completo.pdf-PDFA.pdf?sequence=1 
32. Cabrera G. La macrofauna edáfica como indicador biológico del estado de conservación perturbación del suelo. Resultados obtenidos en Cuba. Pastos y Forrajes 2012; 35(4):349-364. URL Disponible en: http://scielo.sld.cu/ scielo.php?script=sci_arttext\&pid=S0864-03942012000400001

33. Barros E, Mathieu J, Tapia-Coral S, Nascimento ARL, Lavelle, P. Comunidade da macrofauna do solo na Amazônia brasileira. En: Moreira FMS, Siqueira JO Brussaard L. Editores. Biodiversidade do solo em ecossistemas brasileiros. Lavras: UFLA. 171-191. 2008.

Los autores permiten a RECIA reimprimir el material publicado en él. En caso de que un autor quiera traducir o usar una publicación parcial o completa de nuestro Diario, el autor debe obtener un permiso por escrito del editor de la revista.

CC BY 